\title{
Potential role of biochemical placentation markers - pregnancy associated plasma protein-A and human chorionic gonadotropin for early gestational diabetes screening - a pilot study
}

\author{
Vesselina Evtimova Yanachkova'10, Radiana Staynova2 ${ }^{2}$, Ivan Bochev ${ }^{1,3}$, Zdravko Kamenov 4, 5 (i) \\ ${ }^{1}$ Department of Endocrinology, Specialized Hospital for Active Treatment of Obstetrics and Gynaecology "Dr Shterev", \\ Sofia, Bulgaria \\ ${ }^{2}$ Department of Pharmaceutical Sciences, Faculty of Pharmacy, Medical University of Plovdiv, Bulgaria \\ ${ }^{3}$ Department of Molecular Immunology, Institute of Biology and Immunology of Reproduction, Bulgarian Academy of Sciences, \\ Sofia, Bulgaria \\ ${ }^{4}$ Department of Internal Medicine, Medical University Sofia, Bulgaria \\ ${ }^{5}$ Clinic of Endocrinology, University Hospital, Sofia, Bulgaria
}

\begin{abstract}
Objectives: Gestational diabetes mellitus (GDM) is one of the most common pregnancy complications. The universal screening for GDM is usually performed between 24-28 weeks' gestation. This often delays the diagnosis and could increase the risk of adverse pregnancy outcomes. Some of the biochemical placental markers - pregnancy associated plasma protein A (PAPP-A) and free- $\beta$ human chorionic gonadotropin ( $\mathrm{hCG}$ ), probably could provide a diagnostic value for GDM. The aim of our study was to assess if PAPP-A and hCG values were different among pregnant women with and without GDM and respectively, to tested their place in the early GDM screening.

Material and methods: We conducted a retrospective, case-control study by reviewing the clinical database records of 662 pregnant women. The analysis includes the data for a two-year period. The patients included in the observation were divided into two groups - GDM group $(n=412)$ and Euglycemic group $(n=250)$. Early screening for GDM between 9-12 weeks' gestation was performed in 173 of the women in the interventional group due to: registered fasting plasma glucose (FPG) above $5.1 \mathrm{mmol} / \mathrm{L}$, obesity, macrosomia in previous pregnancies or family history for diabetes mellitus. The remaining 239 women underwent universal screening at 24-28 weeks' gestation. Mean serum levels of PAPP-A, hCG, FPG, and body mass index (BMI) were measured between 10-13 gestational weeks. Serum levels of PAPP-A and hCG are presented as multiples of the normal median (MoM), adjusted by maternal baseline characteristics and demographics.

Results: In patients who developed GDM during pregnancy, compared with the control group, we have found significantly lower MoM values of PAPP-A ( $p<0.0001)$, higher levels of FPG $(p<0.0001)$ and higher BMI $(p<0.0001)$. Median hCG MoM was similar in both group of pregnant women.
\end{abstract}

Conclusions: Our findings suggest that low-normal to low reference range values of PAPP-A might be associated with higher risk for GDM. PAAP-A levels can be used as an additional factor to recommend early screening for GDM.

Key words: gestational diabetes mellitus; PAPP-A; early screening

Ginekologia Polska 2022; 93, 5: 405-409

\section{INTRODUCTION}

Gestational diabetes mellitus (GDM) is the most common cause of hyperglycemia during pregnancy. GDM is defined as "any degree of glucose intolerance with onset or first recognition during pregnancy», despite the fact that the state of dysglycemia may exist before conception and continue after birth [1-4]. Current evidence shows that the prevalence of GDM is rising worldwide. In 2019, one in six births was affected by GDM [4]. More than $50 \%$ of women with previous GDM will develop type 2 diabetes mellitus (T2DM) in the first five years after delivery [3-6].

\section{Corresponding author:}

Vesselina Evtimova Yanachkova

Department of Endocrinology, Specialized Hospital for Active Treatment of Obstetrics and Gynaecology "Dr Shterev”, Sofia, Bulgaria

e-mail:v_ess@abv.bg 
A number of risk factors are associated with GDM, including advanced maternal age ( $\geq 35$ yrs.), ethnicity, family history of diabetes, parity $>1$, overweight and obesity, polycystic ovary syndrome, previously delivering a neonate weighing more than $4 \mathrm{~kg}$, etc. [1-4].

GDM is the main risk factor for maternal and fetal complications. GDM significantly increases the risk of hypertensive disorders (gestational hypertension, preeclampsia), caesarean deliveries and birth trauma. Women with GDM are also at higher risk of developing subsequent T2DM. In addition, women who have had GDM with one pregnancy are $30-69 \%$ more likely to develop GDM in subsequent pregnancies [7-12]. Continuous high blood glucose levels can lead to increased fetal and neonatal complications: large for gestational age infants, birth injuries, hypoglycemia, polycythemia, and hyperbilirubinemia. Infants born from women with GDM may suffer from more congenital abnormalities and have a greater propensity of developing neonatal hypoglycaemia, and T2DM later in life [6-8].

The universal screening for GDM, which is performed between 24-28 weeks'gestation, postpones the diagnosis and very often is made after there are pre-existing complications [8,9]. This raises the question if there are markers that are routinely tested in all pregnant women in early pregnancy and could they be a sufficient criteria for recommending early GDM screening. Several placental markers are usually used for aneuploidies screening. These are human chorionic gonadotropin (hCG), placental growth factor (PLGF), soluble fms-like tyrosine kinase 1 (s-fIT-1) and pregnancy-associated plasma protein-A (PAPP-A). If some of these placental factors (hCG, PIGF, s-fTT-1) may be associated with the occurrence of preeclampsia during pregnancy, there is evidence that PAPP-A may also be relevant to carbohydrate disorders manifested during pregnancy.

\section{Aim}

To assess if PAPP-A and $\mathrm{hCG}$ values are different among pregnant women with and without GDM and to propose their potential value in the early GDM screening.

\section{MATERIAL AND METHODS Study design}

A retrospective, case-control, observational study was conducted by reviewing the clinical database records of pregnant women that attended the Specialized Hospital of Obstetrics and Gynecology "Doctor Shterev", Sofia, Bulgaria between December 2017-December 2019.

The study has been considered by Institutional Review Board of the Specialized Hospital of Obstetrics and Gynecology "Doctor Shterev", Sofia Bulgaria. The research project has been conducted in accordance with ethics and law standards for medical research, as stated in active national legislation and Declaration of Helsinki. Proposed non- -interventional retrospective database does not jeopardize confidentiality and autonomy of any patients.

\section{Data collection and measurements}

In this retrospective study, we extracted and analyzed the data from clinical records of 662 pregnant women, of whom 412 were diagnosed with GDM and remaining 250 were euglycemic (control group). Only singleton pregnant women were included in the analysis. Pregnant women with pre-existing diabetes or multiple gestation and those aged less than 18 and higher than 40 years, were excluded from the study. The diagnosis of GDM was confirmed by the 2-hours 75-g oral glucose tolerance test (OGTT) using the diagnostic criteria of the International Association of the Diabetes and Pregnancy Study Groups.

The maternal characteristics considered were age, racial origin (Caucasian), body mass index (BMI), parity, method of conception, previous macrosomia, family history of diabetes mellitus.

Early screening for GDM between 9-12 weeks' gestation was performed in 173 of the women in the intervention group due to registered fasting blood sugar above $5.1 \mathrm{mmol} / \mathrm{L}$, obesity, data for fetal macrosomia in previous pregnancies or family history for diabetes mellitus. The remaining 239 women underwent universal screening at 24-28 weeks' gestation.

Mean serum levels of PAPP-A, hCG, FPG and BMI were measured between 10-13 weeks of gestation. Serum levels of PAPP-A and hCG were tested in the period 11-13 weeks of gestation in favor of screening for aneuploidies. Their levels were determined by electro-chemiluminescent method ECLIA (Cobas 8000, Roche) and were presented as multiples of the median (MoM) after correction based on maternal characteristics (Software provided by the Fetal Medicine Foundation London). FPG values were determined by hexokinase method (Cobas 8000 , Roche) and were presented in $\mathrm{mmol} / \mathrm{L}$ (reference range 3.9-6.1 mmol/L).

\section{Statistics}

The statistical analysis was processed using the SPSS software version 19. We have applied the following statistical methods: descriptive statistics, Kolmogorov-Smirnov test for normality, Mann-Whitney non-parametric test (for comparing outcomes between two independent groups), Pearson chi-square test and Fisher's exact test (for analysing the categorical variables) and receiver operated characteristic (ROC) analysis. Data are presented as mean $\pm S D$, median (IQR) or $\mathrm{n}(\%)$. A p-value less than 0.05 was consider significant.

\section{RESULTS}

Table 1 summarizes the maternal and clinical characteristics of the two groups of patients. Women with GDM and controls were not statistically different in terms 
Table 1. Characteristics of observed pregnant women and biochemical markers

\begin{tabular}{|c|c|c|c|}
\hline Characteristics & GDM group $(n=412)$ & Control group $(n=250)$ & p-value \\
\hline $\begin{array}{l}\text { Maternal age [years], } \\
\text { mean (SD) }\end{array}$ & $33.3(4.9)$ & $32.8(4.3)$ & 0.251 \\
\hline BMI [kg/m²], mean (SD) & $26.08(5.35)$ & $22.9(5.82)$ & 0.422 \\
\hline $\begin{array}{l}\text { Conception method, \% ( } \mathrm{n}) \\
\text { Spontaneous } \\
\text { ART }\end{array}$ & $\begin{array}{l}56(231) \\
44(181)\end{array}$ & $\begin{array}{l}76.8(192) \\
23.2(58)\end{array}$ & 0.0006 \\
\hline Fasting plasma glucose [mmol/L], mean (SD) & $5.48(0.80)$ & $4.8(0.68)$ & $<0.0001$ \\
\hline $\begin{array}{l}\text { Family history of diabetes, \% ( } \mathrm{n} \text { ) } \\
\text { Yes } \\
\text { No }\end{array}$ & $\begin{array}{l}57.8(238) \\
42.2(174)\end{array}$ & $\begin{array}{c}14.8(37) \\
85.2(213)\end{array}$ & $<0.0001$ \\
\hline Previous macrosomia, \% (n) & $10.2(42)$ & $0(0)$ & $<0.0001$ \\
\hline $\begin{array}{l}\text { Parity, \% (n) } \\
\text { Nulliparous } \\
\text { Multiparous }\end{array}$ & $\begin{array}{l}69.4(286) \\
30.6(126)\end{array}$ & $\begin{array}{l}71.2(178) \\
32.8(82)\end{array}$ & 0.459 \\
\hline PAPP-A, adjusted MoM (SD) & $1.2(0.62)$ & $1.3(0.65)$ & $<0.0001$ \\
\hline Free $\beta$-hCG, adjusted MoM & 1.29 & 1.36 & 0.325 \\
\hline $\begin{array}{l}\text { Pharmacological treatment need (metformin or insulin), \% (n) } \\
\text { Yes } \\
\text { No }\end{array}$ & $\begin{array}{l}35.2(145) \\
64.8(267)\end{array}$ & Not applicable & - \\
\hline
\end{tabular}

GDM — gestational diabetes mellitus; SD — standard deviation; BMI — body mass index; assisted reproductive technologies; PAPP-A — pregnancy associated plasma protein $\mathrm{A} ; \mathrm{MoM}$ - multiples of the normal median

of age and BMI. Statistically significant differences were identified regarding family history of diabetes $(p<0.001)$ and previous macrosomia $(p<0.0001)$ (Tab. 1). No statistically significant differences in parity $(p=0.459)$, were found. The median PAPP-A adjusted MoM was significantly lower in GDM group compared to the control group (1.2 vs 1.3; $\mathrm{p}<0.0001)$.

In some of the patients in GDM group due to inadequate blood glucose control on the background of diet treatment, pharmacological therapy with either metformin or insulin was initiated. Patients with GDM who required pharmacological treatment had lower protease levels - PAPP-A0.7 MoM, than those treated with diet alone - 1.01 MoM.

Cases and controls were not statistically different in term of Free $\beta$-hCG adjusted MoM (1.29 vs 1.36; $p=0.325$ ).

Sensitivity and specificity of PAAP-A as a potential diagnostic tool were analyzed using ROC analysis (Fig. 1). The area under the curve (AUC) for the diagnosis of GDM was 0.57 ( $\mathrm{AUC}=0.57, \mathrm{SE}=0.02,95 \% \mathrm{Cl} 0.55-0.61$ ). The cutoff value of the corrected PAPP-A - 0.93 MoM can exclude GDM with a sensitivity level of $43.49 \%$ and a specificity of $69.85 \%$.

\section{DISCUSSION}

It is a known that some of the placental factors, including PAPP-A may be associated with the occurrence of preeclampsia. There is evidence that PAPP-A could also be relevant to carbohydrate disorders manifested during pregnancy.

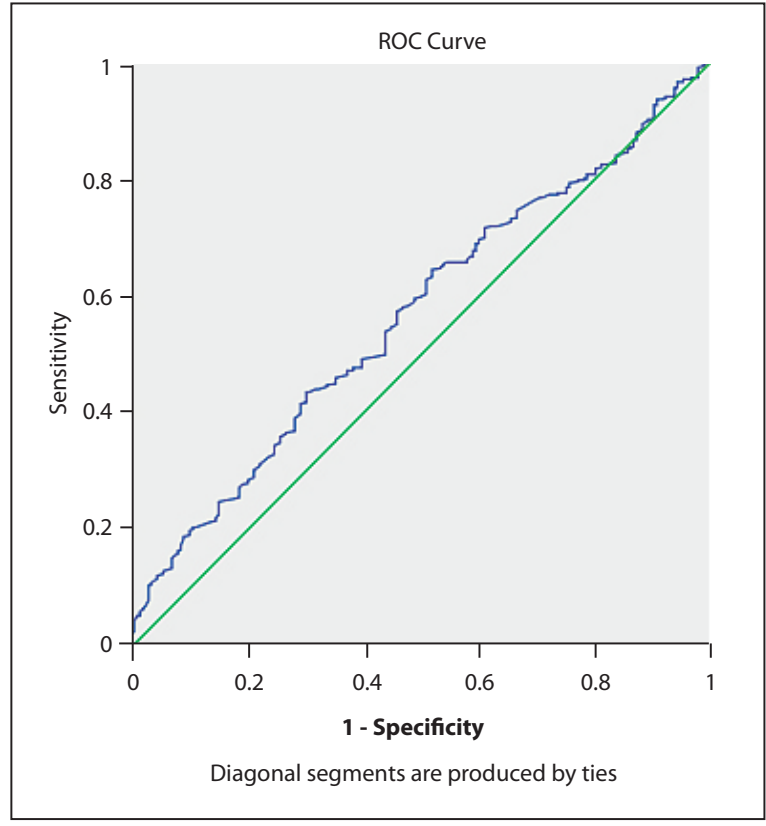

Figure 1. Receiver operating characteristic (ROC) curve demonstrating specificity and sensitivity of pregnancy associated plasma protein $\mathrm{A}$

Our findings allow the role of biochemical placentation markers - pregnancy-associated plasma protein-A for early gestational diabetes screening to be understood.

PAPP-A is a zinc-containing metalloproteinase, first described in 1974 as a protein derived from the placenta of 
pregnant women. It is produced by trophoblast and is detectable in maternal blood from the $28^{\text {th }}$ day of conception. In addition to the placenta, this protein is also expressed in: testis, kidney, colon, ovary (follicular fluid and granulosa cells), fibroblasts, healing after injuries skin, smooth muscle cells on the vessel walls. It is a protease that cleaves the molecule of insulin-like growth factor-bound proteins (IGFBP) $-2,4,5$. This helps the bioactivity of the insulin-like growth factor (IGF) to be improved. PAPP-A can act as an autocrine/paracrine regulator of IGF action. IGF-binding proteins are specific transport proteins that bind IGF1 and IGF-2, thereby blocking their interaction with surface cell receptors. Because PAPP-A cleaves the IGFBP molecule, when its level is reduced, higher levels of IGFBP and low levels of free IGF are observed [14-16].

IGF has role in the regulation of fetal growth (glucose and amino acid uptake); in the autocrine and paracrine control of trophoblast invasion. IGF is a stimulator of protein synthesis in muscles. It also stimulates the utilization of free fatty acids. Its action is regulated by IGFBP. In patients with obesity or diabetes, there is a dysregulation in the secretion of IGFBP, leading to variations in the concentration of IGF-I and IGF-II, as well as their action [13].

Studies have shown that IGFBP-5 in adipocytes and the PAPP-A protease may be relevant to the onset of gestational diabetes mellitus by altering the regulation of functional IGF levels, fat stores, and metabolism. [14-16].

In an uncomplicated pregnancy, the activation of IGFBP-5 increases the levels of insulated IGF-1, IGF-2, and PAPP-A downgrade IGFBP-5. The result is releasing of insulin-like growth factors. This promotes hyperplastic enlargement of adipocytes and angiogenesis. In women with GDM, there is a reduced bioavailability of IGF due to inadequate levels of IGFBP-5 and likely decreased levels of PAPP-A. The result is inadequate angiogenesis, resulting in adipocyte hypertrophy and decreased capillary density. Low-grade inflammation, lipotoxicity respectively, leading to insulin resistance and impaired carbohydrate tolerance have been observed [14-16].

The current study indicates that women with GDM have low or low normal PAAP-A levels. Our findings confirmed the results from previous studies [16-21]. There are conflicting data regarding the relationship between PAPP-A levels and the manifestation of gestational diabetes. However, studies could not determine definitively if low PAPP-A levels lead to carbohydrate metabolism disorders. [20, 21].

As PAPP-A is a marker that is routinely tested in $80 \%$ of pregnant women, it could be used as an early marker for diagnosis or at least referral to a GDM test. We observed lower serum levels of PAPP-A in women with GDM as well as other research teams. In our study pregnancy-associated plasma protein-A (PAPP-A) levels below 0.9 MoM were as- sociated with increased prevalence of GDM. In addition to the fact that low levels of PAPP-A can serve as predictors of carbohydrate disorders, our observations show that the lower the levels of protease, the greater the likelihood of the need for the addition of pharmacological therapy in patients with already established GDM.

\section{CONCLUSIONS}

Early diagnosis of GDM is crucial in order to avoid complications during the course of pregnancy. In this study PAAP-A levels below 0.9 MoM were associated with increased prevalence of GDM for the Bulgarian pregnant women. As PAPP-A is routinely tested in the period 11-13 weeks of gestation in favor of screening for aneuploidies, it could be referral to an early GDM test. Further studies are needed to accept the possibility of using certain placental markers as an alternative for a recommendation for GDM screening. However, low and low-normal PAAP-A levels can be used as an additional factor to recommend early screening for GDM.

\section{Conflict of interest}

The authors declare there is no conflict of interests involved in elaborating and publishing this original article.

\section{REFERENCES}

1. 2. Classification and Diagnosis of Diabetes: Standards of Medical Care in Diabetes-2020. Diabetes Care. 2019; 43(Supplement 1): S14-S31, doi: $10.2337 / \mathrm{dc} 20$-s002.

2. Bulgarian Society of Endocrinology. Recommendations for good clinical practice for Diabetes Mellitus. Bulgarian Society of Endocrinology, Sofia 2019

3. Todorova K. Diabetes and pregnancy. 1st. Artik, Sofia 2010.

4. International Diabetes Federation. IDF Diabetes Atlas, 9th ed. International Diabetes Federation, Brussels 2019.

5. Feig DS, Berger H, Donovan L, et al. Diabetes Canada Clinical Practice Guidelines Expert Committee. Diabetes and Pregnancy. Can J Diabetes. 2018; 42 Suppl 1: S255-S282, doi: 10.1016/j.jcjd.2017.10.038, indexed in Pubmed: 29650105.

6. Hod M, Kapur A, Sacks DA, et al. The International Federation of Gynecology and Obstetrics (FIGO) Initiative on gestational diabetes mellitus: A pragmatic guide for diagnosis, management, and care. Int J Gynaecol Obstet. 2015; 131 Suppl 3: S173-S211, doi: 10.1016/S00207292(15)30033-3, indexed in Pubmed: 29644654.

7. Bodmer-Roy S, Morin L, Cousineau J, et al. Pregnancy outcomes in women with and without gestational diabetes mellitus according to the International Association of the Diabetes and Pregnancy Study Groups criteria. Obstet Gynecol. 2012; 120(4): 746-752, doi: 10.1097/AOG.0b013e31826994ec, indexed in Pubmed: 22996090.

8. Zhang X, Xu H, Hu R, et al. Gestational diabetes mellitus: prevalence, risk factors, maternal and infant outcomes. Int J Gynaecol Obstet. 2001; 75(3): 221-228, doi: 10.1016/s0020-7292(01)00496-9, indexed in Pubmed: 11728481.

9. Davey RX, Hamblin PS. Selective versus universal screening for gestational diabetes mellitus: an evaluation of predictive risk factors. Med J Aust. 2001;174(3): 118-121, doi: 10.5694/j.1326-5377.2001.tb143181.x, indexed in Pubmed: 11247613.

10. Lee KW, Ching SM, Ramachandran V, et al. Prevalence and risk factors of gestational diabetes mellitus in Asia: a systematic review and meta-analysis. BMC Pregnancy Childbirth. 2018; 18(1): 494, doi: 10.1186/s12884018-2131-4, indexed in Pubmed: 30547769.

11. Svare JA, Hansen BB, Mølsted-Pedersen L. Perinatal complications in women with gestational diabetes mellitus. Acta Obstet Gynecol Scand. 2001;80(10):899-904, doi: 10.1034/j.1600-0412.2001.801006.x, indexed in Pubmed: 11580734 
12. Lalooha F, Elmizadeh $\mathrm{K}$, Javadi $\mathrm{A}$, et al. Association between abnormal glucose challenge test and pregnancy outcome. J Zanjan Univ Med Sci. 2012; 20(83): 53-61.

13. Clemmons DR. Metabolic actions of insulin-like growth factor-l in normal physiology and diabetes. Endocrinol Metab Clin North Am. 2012; 41(2): 425-43, vii, doi: 10.1016/j.ecl.2012.04.017, indexed in Pubmed: 22682639.

14. Bayes-Genis A, Conover CA, Overgaard MT, et al. Pregnancy-associated plasma protein $\mathrm{A}$ as a marker of acute coronary syndromes. $\mathrm{N}$ Engl J Med. 2001; 345(14): 1022-1029, doi: 10.1056/NEJMoa003147, indexed in Pubmed: 11586954.

15. Rojas-Rodriguez R, Lifshitz LM, Bellve KD, et al. Human adipose tissue expansion in pregnancy is impaired in gestational diabetes mellitus. Diabetologia. 2015; 58(9): 2106-2114, doi: 10.1007/s00125-015-3662-0, indexed in Pubmed: 26067361.

16. Saruhan Z, Ozekinci $M$, Simsek $M$, et al. Association of first trimester low PAPP-A levels with adverse pregnancy outcomes. Clin Exp Obstet Gynecol. 2012; 39(2): 225-228, indexed in Pubmed: 22905470.

17. Lovati $E$, Beneventi $F$, Simonetta $M$, et al. Gestational diabetes mellitus: including serum pregnancy-associated plasma protein-A testing in the clinical management of primiparous women? A case-control study. Diabetes Res Clin Pract. 2013; 100(3): 340-347, doi: 10.1016/j. diabres.2013.04.002, indexed in Pubmed: 23642968.

18. Syngelaki A, Kotecha R, Pastides A, et al. First-trimester biochemical markers of placentation in screening for gestational diabetes mellitus. Metabolism. 2015; 64(11): 1485-1489, doi: 10.1016/j.metabol.2015.07.015, indexed in Pubmed: 26362726.

19. Nanda S, Savvidou M, Syngelaki A, et al. Prediction of gestational diabetes mellitus by maternal factors and biomarkers at 11 to 13 weeks. Prenat Diagn. 2011; 31(2): 135-141, doi: 10.1002/pd.2636, indexed in Pubmed: 21268030.

20. Talasaz ZH, Sadeghi R, Askari F, et al. First trimesters Pregnancy-Associated Plasma Protein-A levels value to Predict Gestational diabetes Mellitus: A systematic review and meta-analysis of the literature. Taiwan J Obstet Gynecol. 2018; 57(2): 181-189, doi: 10.1016/j.tjog.2018.02.003, indexed in Pubmed: 29673658.

21. Donovan BM, Nidey NL, Jasper EA, et al. First trimester prenatal screening biomarkers and gestational diabetes mellitus: A systematic review and meta-analysis. PLoS One. 2018; 13(7): e0201319, doi: 10.1371/journal. pone.0201319, indexed in Pubmed: 30048548 